\title{
CLINICAL ELECTRO-ENCEPHALOGRAPHY. I
}

The electro-encephalogram is a record of the constantly changing small electrical potentials from the brain. These potentials are picked up from the scalp by closely-applied electrodes and are then amplified and written out as an ink trace.

\section{The Electrodes}

These fall into three main classes: pad electrodes held in place by a head-cap consisting of strips of rubber used for routine recording; stick-on electrodes glued in place with collodion, particularly employed for sleep recordings and restless or young subjects; and needle electrodes which, after sterilization, are inserted into the scalp, required for emergency recording from unconscious patients when speed is essential. Longer needle electrodes are needed for sphenoidal recordings in patients with suspected temporal lobe epilepsy. To obtain good electrical contact with the scalp, saline jelly is employed with pad and stick-on electrodes, the acceptable resistance between a pair of electrodes is then usually $5,000 \mathrm{ohms}$ or less.

Twenty electrodes are usually used and are placed in positions determined by the internationally recognized "ten-twenty system". This employs standard bony landmarks (e.g. nasion and inion) as reference points, so that the relationship of the electrodes to the underlying cortex is known. The electrodes are thus labelled, frontal, parietal, etc., and when they are accurately placed repeat recordings can be assessed with precision even when the skull is growing as in a child.

\section{The EEG Machine and Recording Conventions}

The EEG machine is essentially an electronic device, now constructed with transistors, capable of amplifying the minute brain potentials about a million times. The machine consists of eight or sixteen independent channels, each of which comprises an input, an amplifier, and an output. A series of switches alters the circuits so that: (1) the height or amplitude of the trace may be changed by the "gain control", the level for recording being usually 50 or $100 \mu \mathrm{v}$. per cm.; (2) unwanted components can be removed, low frequencies by the "time constant" and high frequencies by the "H.F. filter". There are also selector switches which change the combinations of electrodes from which recordings are made. These are known as "montages" and are usually symmetrical transverse or longitudinal lines on the head. The output voltage drives a pen which writes directly on a moving strip of paper pulled through the machine at a constant rate by an electric motor (routinely at $3 \mathrm{~cm} . / \mathrm{sec}$.). Recordings are carried out by one of two main methods:

(1) The bipolar method, in which potentials from two neighbouring electrodes are fed into one channel of the EEG machine, and the circuit is so arranged that potential differences between the two brain areas are amplified;

(2) The mono-polar or reference method, in which the potential of each electrode is compared with a common reference point as near zero potential as possible (the ear-lobe, or the averaged potential of all electrodes).

The following convention of EEG recording is employed. When the upper input of a channel is affected by a positive potential relative to the lower input, the pen deflection on the paper trace is downwards; if the upper input is affected by a negative potential the pen moves upwards. Potential changes at the lower input have an opposite effect. This arrangement in bipolar linkages leads to an electrical localizing sign known as "phase reversal".

\section{Clinical Procedure}

The EEG takes about 30 minutes to perform. For most of the time the patient is relaxed on a couch, with eyes closed to reduce eye movements and other artefacts. The eyes are opened for standard periods to test the effect on brain activity. Two other techniques are used routinely as these may produce physiological changes as well as elicit abnormalities not seen in the resting record:

(1) Hyperventilation, a 3-minute period of which may produce a mild respiratory alkalosis;

(2) Photic stimulation using a stroboscope to deliver brief (to preclude retinal damage) bright $(80,000$ candles) repetitive flashes at rates from 1 to 30 per sec. 
Other forms of activation are employed, the most common being sleep. This may occur spontaneously or be induced by oral or intravenous barbiturates.

\section{Difficulties}

(1) Extracranial structures produce larger potentials than the brain (e.g. eye movements and contraction of the facial or temporal muscles). Sweating will also lead to gross artefacts. Patients must have the test explained to reduce tension, and they must lie as still as possible.

(2) Unsatisfactory electrode attachment may cause large and troublesome artefacts, hence care is needed in their attachment.

(3) Changes in the state of the patient produce alterations in the EEG. Some like drowsiness are easy to monitor, others like metabolic variations during the menstrual cycle are more difficult. Alterations in medication and seizure frequency are particularly important variables.

\section{Physiological Basis of the EEG}

The main assumption is that the potentials recorded from the scalp reflect the changes in the underlying neurones. It is known that a dead brain does not emit any potentials; their exact origin, however, is uncertain. They are quite different from the brief action potentials of neurones which last only a millisecond; the period of the alpha wave is about 100 times longer. Waxing and waning of biochemical rather than neural processes or a combination of reverberating neural circuits have been suggested to explain the spontaneous wave-like alpha activity, but so far these problems are unsolved.

Features of the Normal and Abnormal EEG (Fig. 1)

The normal EEG consists of a complex ever-changing pattern of electrical potentials, the frequency and the amplitude of the waves varying. In the normal adult EEG, two components stand out, although automatic frequency analysis has shown a more complex pattern. First, the alpha rhythm, with a frequency of 8-14 c/sec. varying in amplitude from $10-100 \mu \mathrm{v}$, is located over the post-

\begin{tabular}{|c|c|c|}
\hline Type of Activity & Wave Form and Frequency & Remarks \\
\hline Alpha & $8-14 c / s e c$ & $\begin{array}{l}\text { Normal activity seen in } \\
\text { parieto-occipital region } \\
\text { "Blocked" by eye opening }\end{array}$ \\
\hline Beta & above $14 \mathrm{c} / \mathrm{sec}$ & $\begin{array}{l}\text { Normal activity } \\
\text { Marked increase after drug } \\
\text { administration especially } \\
\text { Barbiturate }\end{array}$ \\
\hline Theta & 4-7 c/sec. & $\begin{array}{l}\text { Occurs in drowsiness, } \\
\text { youth, and abnormally in } \\
\text { many conditions }\end{array}$ \\
\hline Delta & . & $\begin{array}{l}\text { Occurs in deep sleep in } \\
\text { normal subjects, also in } \\
\text { coma and many disorders }\end{array}$ \\
\hline
\end{tabular}
erior head regions.

This activity is seen during mental relaxation with the eyes closed and is attenuated when the eyes are opened. Second, the beta rhythm, with a frequency of $15-30 \mathrm{c} / \mathrm{sec}$. and an amplitude of 5-10 $\mu \mathrm{v}$, is most obvious anteriorly and is unaffected by eye opening. Theta activity, with a frequency range of 4-7 c/sec., is not seen in normal adults except in a state of drowsiness; it is, however, a normal feature in children and adolescents. Delta waves, with a frequency of $\frac{1}{2}-4 \mathrm{c} / \mathrm{sec}$., occur during deep sleep in normal adults and younger subjects, but characterize the EEG of pathological conditions such as cerebral tumour in waking patients.

(to be concluded)

Donald F. Scott, Department of Electro-encephalography, The London Hospital, London, E.1. 